\title{
CARTOGRAFÍA DE ZONAS PROBABLES DE ALUDES EN EL VALLE DE ORDESA (PIRINEO ARAGONÉS)
}

\author{
Asunción JULIÁN y Javier CHUECA \\ Departamento de Geografía y Ordenación del Territorio \\ Universidad de Zaragoza
}

\begin{abstract}
Resumen: En este trabajo presentamos una cartografía de zonas probables de aludes en el valle de Ordesa en la que se incluyen todas aquellas áreas que se han visto o que pueden verse afectadas por avalanchas de nieve. La metodología utilizada, en la que se combina la observación de fotogramas aéreos con el trabajo de campo y, en menor medida la encuesta sobre el terreno, se basa en la consideración de aquellos factores intrínsecos a las vertientes como son el límite altitudinal, el valor de las pendientes, la morfología y rugosidad de las laderas, su orientación y la cubierta vegetal presente en la zona.
\end{abstract}

Palabras clave: Cartografía, aludes, valle de Ordesa, Pirineo Aragonés.

\begin{abstract}
In this work a cartography of probables snow avalanches areas in the Ordesa valley is showed. In this cartography we include all the areas which have been or could be afectted by some snow avalanches. The methodology adopted combines the observation of aerial photographies with field work and, to a lesser extent, the use of enquiries in the area, and it is based on the consideration of those factors intrinsicly related to the slopes like altitudinal limits, the slope values, the morphology and rugosity of the versants, the aspect of the slopes and the vegetal cover.
\end{abstract}

Key words: Cartography, avalanches, Ordesa valley, Aragonese Pyrenees

\section{INTRODUCCIÓN}

Las avalanchas de nieve constituyen un fenómeno frecuente dentro de un país montañoso como el nuestro. Aunque las cifras de muertes por aludes no alcanzan los niveles de las registradas en otros lugares del entorno europeo como Austria, Suiza o Francia, no por ello deben despreciarse, y si a los costes personales sumamos los considerables costes directos e indirectos ligados a la afección de las infraestructuras, debe- 
mos contemplar el riesgo de avalanchas como importante. Este riesgo viene, sin duda, además potenciado por el auge que las actividades de montaña registran en estas últimas décadas.

El sistema montañoso español que padece el mayor número de avalanchas es el pirenaico, con una clara concentración en los sectores aragonés y catalán. Lamentablemente, hasta el momento actual, - y a diferencia de lo que ocurre en la comunidad vecina donde existe una ya consolidada trayectoria de investigación en relación con los aludes-, en Aragón los trabajos en los que se aborda esta temática son muy escasos y se limitan a breves referencias dentro de trabajos geomorfológicos más amplios o bien a análisis centrados en la evolución del manto nival.

Una de las tareas prioritarias en los estudios sobre riesgo de avalanchas consiste en delimitar aquellas áreas que, considerando diversos factores (topografía, vegetación, exposición, etc.), están expuestas al desencadenamiento de aludes. La cartografía temática que se obtiene como resultado constituye una herramienta básica para la ordenación y planificación del territorio y es el punto de partida para la elaboración de cartografías de riesgos.

Son muchos los ámbitos del Pirineo Aragonés en los que el estudio de los aludes es no sólo un atrayente objetivo de investigación sino una necesidad cada vez más acuciante de cara a la ordenación del espacio. Una de estas zonas, especialmente representativa tanto por la ubicuidad de las avalanchas como por tratarse de un territorio en el que las actividades recreativas ligadas a la montaña alcanzan una gran relevancia, es el valle de Ordesa. En este trabajo analizamos los factores básicos con representación espacial que favorecen o determinan el desencadenamiento de aludes y presentamos una cartografía en la que se delimitan las áreas susceptibles de verse afectadas por dicho fenómeno.

\section{LA ZONA DE ESTUDIO}

El área de estudio se localiza en el Pirineo Central Aragonés y coincide básicamente en su totalidad con el valle de Ordesa, territorio integrado dentro del Parque Nacional de Ordesa y Monte Perdido (Figura 1). Este valle, de dirección fundamental Este-Oeste se halla recorrido por el río Arazas, característico curso de montaña que, tras recibir el drenaje de la vertiente meridional de los elevados macizos de Monte Perdido ( $3.355 \mathrm{~m}$ ), Cilindro $(3.322 \mathrm{~m})$, Marboré $(3.247 \mathrm{~m})$, Taillón $(3.170 \mathrm{~m})$ y Gabietos $(3.334 \mathrm{~m})$, y el mucho más limitado de la vertiente septentrional de la Sierra de las Cutas $(2.236 \mathrm{~m})$ y Custodia $(2.387 \mathrm{~m})$, confluye con el río Ara. Los límites concretos que delimitan el sector cartografíado vienen marcados al Sur por las sierras que acabamos de mencionar, y al Norte por la línea definida por Mondarruego $(2.755 \mathrm{~m})$, Punta Escuzana $(2.847 \mathrm{~m})$, circo de Carriata, Pico de Salarons $(2.752 \mathrm{~m})$, circo de Cotatuero, Tobacor $(2.769 \mathrm{~m})$ y circo de Soaso. 


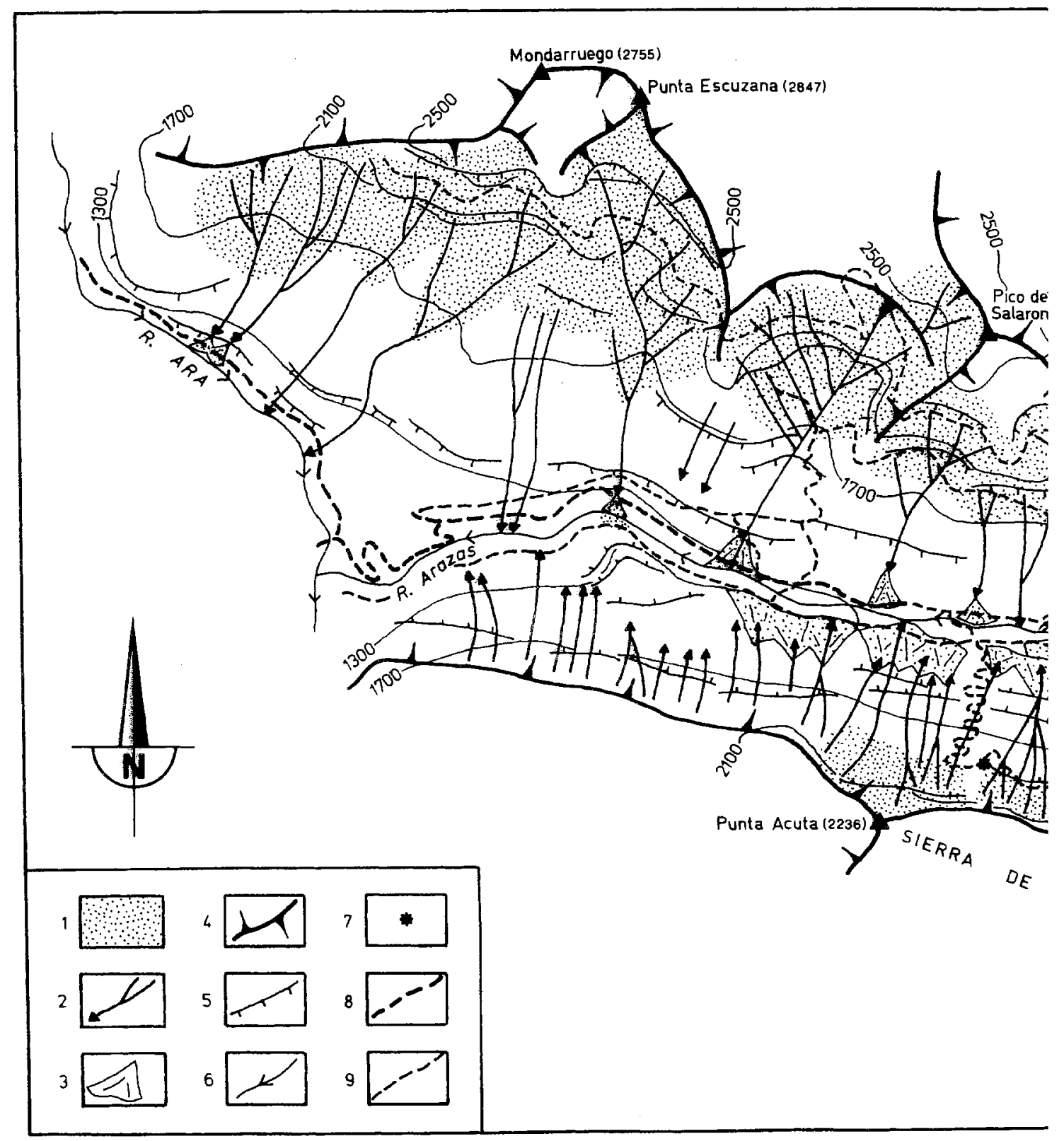

Figura 1. Cartografía de zonas probables de aludes (valle de Ordesa). Leyenda: 1 (de aludes, torrenciales, de gravedad; 4) Límites de valle glaciar y/o । 
La práctica totalidad de este espacio se emplaza por encima de los $1.100 \mathrm{~m}$ de altitud y una buena parte sobrepasa los $1.700 \mathrm{~m}$, aspecto que, como comentaremos más adelante, es fundamental en relación con la innivación. Desde el punto de vista geológico la zona analizada se ubica dentro de las Sierras Interiores pirenaicas, integradas en este sector por una superposición de varios mantos de corrimiento: la Unidad I o Manto de Gavarnie, la Unidad II o Manto de Monte Perdido, y fuera ya de los límites marcados para este trabajo la Unidad III o Escama de Las Tres Marías y la Unidad IV o Escama de las Cumbres (VAN DE VELDE, 1967; RÍOS et al., 1989). Los materiales aflorantes, de naturaleza básicamente calcárea o areniscosa, integran una serie de unidades litológicas que cronológicamente comprenden desde el Cretácico Superior hasta el Eoceno Inferior. La Formación Estrecho, de edad Campaniense y con un claro predominio de calizas, constituye la base de la serie, siendo apenas visible; mayor significación espacial y morfológica tiene la Formación Tozal (Maestrichtiense), calizas arenosas y areniscas de cemento calcáreo, fácilmente identificables por su característica pátina pardo-amarillenta en las que se han labrado buena parte de los escarpes más abruptos del valle de Ordesa. Sobre esta serie se disponen dos formaciones carbonatadas, la Formación Salarons (Paleoceno) y la Formación Gallinero (Eoceno Inferior), ambas muy bien representadas en todo el sector. Situada por encima de estas calizas y dolomías se emplaza una formación de margas, atribuida al Ilerdense Superior, cuya mayor potencia visible corresponde a la Sierra Custodia. Por último, también se hallan ampliamente representados los sedimentos tipo flysch (Formación Flysch), arenis-

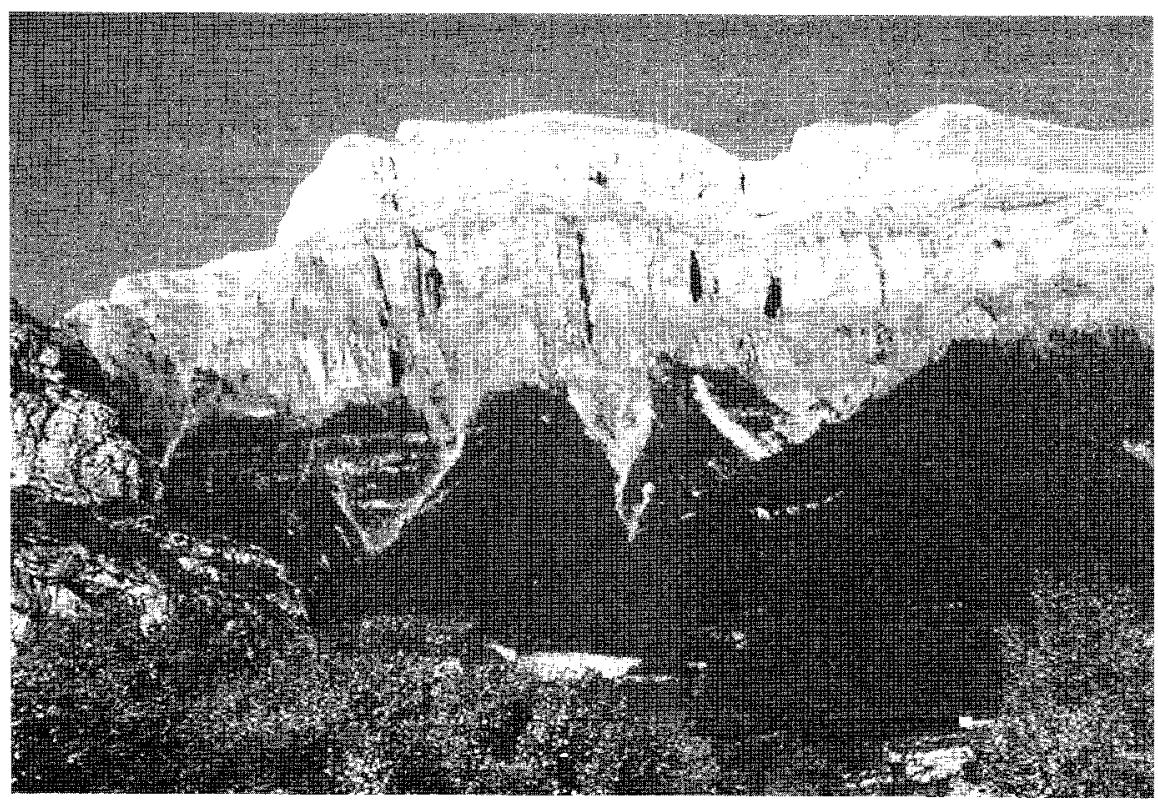

Fotografía 1. Canales de aludes en la entrada del valle de Ordesa (vertiente Mondarruego-Punta Escuzana) 
cas y margas alternantes en capas decimétricas que aparecen entre otros lugares en Punta Escuzana, Salarons, Punta Tobacor o en Sierra Custodia. En cuanto a la disposición que adoptan los materiales de estas formaciones, destaca la acusada horizontalidad de la Formación Tozal, Salarons y Gallinero en la pared Sur del Valle de Ordesa frente a la inflexión que registran estos mismos materiales en la Sierra de las Cutas. La parte superior de la serie integrada por los sedimentos tipo flysch se encuentra fuertemente replegada, especialmente en los sectores de Mondarruego y Salarons.

La configuración del modelado del valle de Ordesa es consecuencia de la naturaleza del roquedo (básicamente calcáreo, aunque también el flysch tiene una importante representación) y de la disposición de los estratos; sin olvidar la importante huella que ha impreso el glaciarismo cuaternario. El cañón de Ordesa, labrado por el progresivo encajamiento del río Arazas sobre las series carbonatadas, presenta espectaculares acantilados en los que quedan en resalte los afloramientos más duros, conformando estrechos escarpes de cierta continuidad; mientras, hacia la parte inferior de las laderas los taludes de derrubios rompen la verticalidad de las paredes. Por el contrario, en aquellos sectores en los que aflora el flysch, los escarpes verticales son reemplazados por relieves alomados de pendientes suavizadas.

Desde el punto de vista climático nos encontramos ante un espacio de alta montaña de influencias atlánticas ya muy debilitadas, lo que se plasma en el régimen y volumen de las

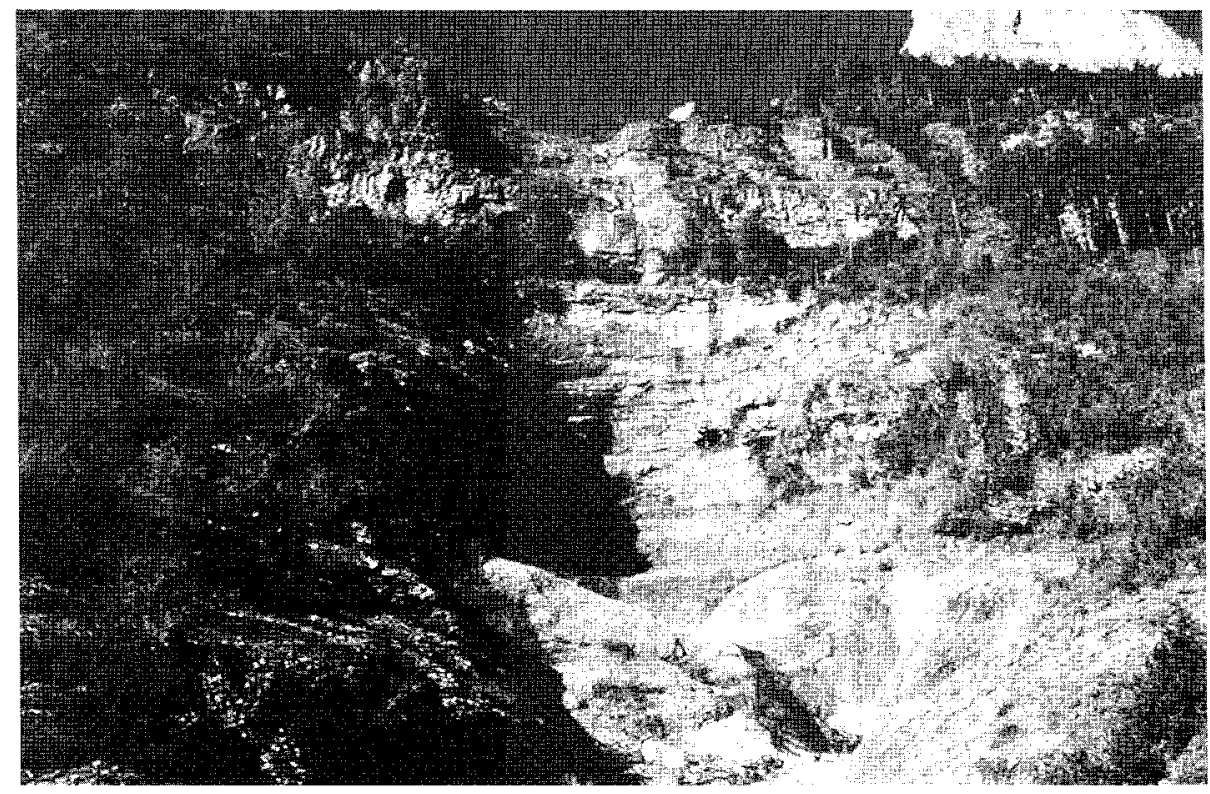

Fotografía 2. Aspecto de la vegetación en un canal de aludes al pie de las Fajas del Mallo (Murallas de la Gatera), próximo al curso del río Ara 
precipitaciones. La estación meteorológica más próxima a la zona de estudio se encuentra en el refugio de Góriz (2.200 m), si bien la serie de años con datos disponibles es muy limitada (BALCELLS y GIL, 1992). GARCÍA-RUIZ y MARTÍ BONO (1993) en su estudio sobre el Parque Nacional de Ordesa y Monte Perdido, a partir de los datos facilitados por esta última estación así como de los procedentes de otras ubicadas ya en la periferia del Parque (Torla, Broto, Linás de Broto, Fanlo, etc.) se refieren a unas precipitaciones relativamente importantes, propias de un área de montaña (entre otros valores podemos citar los de Linás de Broto, a unos $1.330 \mathrm{~m}$ de altura, donde se registran como media anual $1.635 \mathrm{~mm}$ de precipitación; Fanlo, a unos 1.320, con $1.621 \mathrm{~mm}$; Broto, a $1.005 \mathrm{~m}$, con un registro de $1.323 \mathrm{~m}$; o Torla, a $1.053 \mathrm{~m}$ y con $1.424 \mathrm{~mm}$ ). Lógicamente estas cifras deben ser mayores en cotas superiores, de ahí que sorprenda que en la estación de Góriz, a $2.220 \mathrm{~m}$. el valor medio anual durante el período 1981-1989 sea de unos $1.650 \mathrm{~mm}$, circunstancia que BALCELL y GIL (1992) atribuyen a las condiciones de sequía registradas en la década de los ochenta. El mayor volumen de precipitaciones se produce en otoño y primavera, apreciándose un claro descenso en verano e invierno, época en la que buena parte de dichas precipitaciones son de carácter nivoso. Térmicamente, las temperaturas también se hallan estrechamente relacionadas con la altura, lo que significa que van a alcanzar valores muy bajos: así en Broto la temperatura media invernal es de $3,4^{\circ} \mathrm{C}$, en Fanlo $2^{\circ}$, y en Góriz inferior a $\operatorname{los} 0^{\circ}$ a lo largo de tres meses. De acuerdo con los cálculos efectuados por GARCÍARUIZ et al. (1985), la isoterma de $0^{\circ}$ durante la estación fría (noviembre-abril) se localiza a $1.670 \mathrm{~m}$ en la cuenca del Ara, lo que repercute en la potenciación estacional (especialmente a finales de invierno y principios de primavera) de los procesos ligados a la nivación en la zona estudiada (entre éstos los aludes).

En el valle de Ordesa la vegetación natural se conserva en buenas condiciones, lo que permite establecerse un transecto geobotánico-tipo en función de la topografía y la orientación (VILLAR et al., 1997): a partir de los $1.200 \mathrm{~m}$ y hasta los $1.600-1.700 \mathrm{~m}$, dominando las umbrías del cañón, encontramos las hayas (Fagus syluatica) -en ocasiones formando bosques mixtos junto a los abetos (Abies alba) - que también colonizan las solanas en torno a los $1.600 \mathrm{~m}$. Como especie arbórea más significativa de las vertientes de solana destaca el pino silvestre (Pinus sylvestris), siempre por debajo de los $1.800 \mathrm{~m}$, límite a partir del cual pasa a ser sustituido por el pino negro (Pinus uncinata). El pino negro, única especie forestal que se adapta a las bajas temperaturas invernales y a la importante innivación que se registra en estas cotas, coloniza incluso los escarpes de las paredes de Ordesa. Sin embargo, en determinados sectores como en el tramo alto y medio-alto del valle, el límite superior del bosque es artificial y responde a la deforestación que, desde hace ya largos años, se realizó para proporcionar pastos al ganado. En estas áreas, potencialmente forestales, se encuentran las típicas tascas pirenaicas de gramíneas. Por encima de los 2.200$2.300 \mathrm{~m}$ las manchas aisladas de comunidades herbáceas alpinas (pastos de altura, abiertos, a base entre otras de Saponaria caespitosa y Festuca scoparia), van alternándose con el roquedo desnudo, creciendo la extensión de éste conforme nos desplazamos en altura y los suelos se hacen cada vez menos potentes y más inmaduros. 


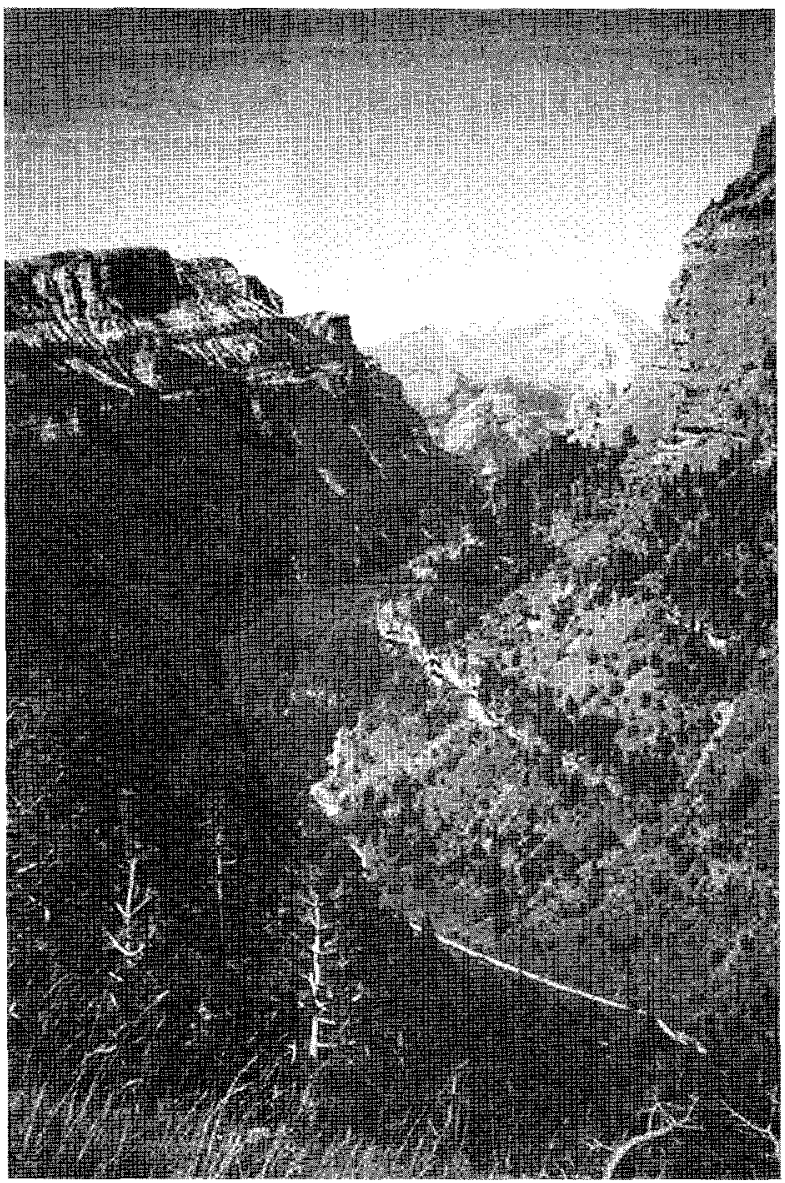

Fotografía 3. Panorámica del valle de Ordesa desde la senda de los Cazadores. Se aprecian zonas probables de aludes y canales en las dos vertientes del valle: a la izquierda bajo la Sierra de las Cutas y a la derecha en las laderas de Punta Tobacor

\section{EL MÉTODO DE TRABAJO}

Los aludes de nieve se engloban dentro de los procesos geomorfológicos característicos que afectan a las laderas de las áreas de montaña. Los factores que intervienen en su desencadenamiento pueden agruparse en dos grandes tipos: factores extrínsecos a las vertientes, vinculados a las condiciones nivo-meteorológicas y, dado que en ellos la variabilidad temporal es muy importante, no representables espacialmente; y factores intrínsecos a las vertientes, localizables sobre el territorio y, por consiguiente, susceptibles de cartografiar. La cartografía de zonas probables de aludes, en la que se reflejan aquellas zonas en 
las que se han producido aludes en algún momento, o en las que considerando diversos criterios podrían desencadenarse, se fundamenta en este segundo tipo de factores.

La metodología adoptada para elaborar la cartografía que aquí presentamos está ya ampliamente difundida en varios trabajos de este tipo. Fue establecida en los años 70 por parte del Ministerio de Agricultura francés (CEMAGREF, 1981), y que en nuestro país ha sido utilizada fundamentalmente por parte del grupo de trabajo de la Universidad de Barcelona (FURDADA, et al., 1989; FURDADA, 1996). En ella se establecen tres fases complementarias que pueden ser simultáneas en el tiempo: la fotointerpretación, la observación directa en el campo, y las encuestas a personas vinculadas a las áreas de montaña objeto de análisis.

Si bien las tres etapas son importantes, y cada una ella cuenta con sus propias ventajas e inconvenientes, la fotointerpretación es básica, pues a través de ella, observando las huellas dejadas en el medio natural, podemos constatar la ocurrencia de avalanchas; pero además, a partir de determinados criterios podemos delimitar aquellas áreas más favorables al desencadenamiento de aludes. En nuestro trabajo hemos utilizado fotografías aéreas verticales, en blanco y negro, a escala 1:30.000, suministradas por el Instituto Geográfico Nacional que fueron tomadas en septiembre de 1981.

El trabajo de campo es imprescindible para obtener información de aquellos sectores afectados por sombras, sectores que en ámbitos con relieves verticalizados, como es el caso del valle de Ordesa, pueden representar una extensión considerable (de hecho, una buena parte de la ladera de orientación Norte ubicada al pie de la Sierra de las Cutas es difícilmente observable en la fotos aéreas). Por otra parte, la observación directa sobre el terreno permite disponer de datos más detallados en relación fundamentalmente con la vegetación (huellas del paso de la nieve, grado de recuperación en función de la frecuencia de aludes); así como actualizar la información obtenida a través de la fotografía aérea, algo que puede ser básico cuando no se dispone de fotogramas relativamente recientes. El reconocimiento de campo que hemos llevada a cabo en el valle de Ordesa nos ha permitido atestiguar el funcionamiento de muchos de los principales caminos de avalanchas e, incluso, en casos puntuales, comprobar cómo el área afectada por un alud de gran magnitud (Bco. de la Canal; campaña invernal 1996-1997) llegó a rebasar los límites máximos esperados a partir de fotointerpretación.

La fase de encuesta puede aportar información muy valiosa de cara a ratificar los datos obtenidos en las etapas anteriores y, especialmente, en relación con la dinámica de los aludes. Lamentablemente, acometer una campaña de encuestas, sobre todo si se pretende abordar de forma sistemática entre los distintos grupos de población relacionados con la montaña y dotarla de una cierta continuidad a lo largo de varios años, es tarea bastante compleja. Por otra parte, la disposición de determinadas personas a facilitar datos sobre el desencadenamiento de avalanchas puede estar limitada por condicionantes económicos, apreciándose frecuentemente cierta reticencia a hablar de un tema que tiene «mala prensa». Para la realización de este trabajo no hemos llevado a cabo una encuesta sistemática, pero sí que hemos corro- 
borado algunos datos significativos con la colaboración del Centro de Interpretación del Parque Nacional de Ordesa y Monte Perdido y con representantes del colectivo montañero.

Respecto a los criterios que permiten identificar y delimitar las zonas probables de aludes tienden a clasificarse en directos e indirectos, en función de que se refieran a los factores intrínsecos a las vertientes, en el primer caso, o a factores extrínsecos, relacionados con las condiciones nivo-meteorológicas, en el segundo caso (FURDADA, 1996; MCCLUNG y SCHAERER, 1996). Para elaborar la cartografía sobre el valle de Ordesa los criterios directos en los que nos hemos apoyado son: el límite altitudinal, el valor de las pendientes, la morfología y rugosidad de las laderas, y la vegetación. Entre los criterios indirectos hemos considerado la orientación de las vertientes respecto a la radiación solar. La combinación coherente de todos, o al menos de buena parte de estos criterios, nos ha permitido plasmar en la cartografía las áreas más expuestas a las avalanchas de nieve.

1. El límite altitudinal marca la cota inferior a partir de la cual durante una determinada época del año (finales de invierno y principios de primavera) las precipitaciones son en forma de nieve y las bajas temperturas permiten su conservación. Este límite viene aproximadamente marcado por la isoterma de $0^{\circ} \mathrm{C}$ que, tal y como comentábamos al referirnos a los rasgos climáticos de la zona, ha sido establecida para la cuenca del Ara en torno a los $1.670 \mathrm{~m}$ (GARCÍA-RUIZ et al., 1985). Una parte importante de la zona de estudio se ubica por encima de esta cota; pero además, hemos de considerar que, si bien en las áreas situadas a alturas inferiores, en principio, la probabilidad de que se desencadene un alud es mínima, sin embargo, esas mismas zonas pueden verse afectadas por el paso o la llegada de una avalancha.

2. Respecto a los valores de pendiente más favorables al desencadenamiento de aludes se considera necesaria una inclinación de al menos $28^{\circ}$ para que la componente longitudinal del peso de la nieve supere las fuerzas de resistencia; en el otro extremo, sobre vertientes con ángulos de pendiente superiores a los $45^{\circ}$, la cantidad de nieve que se llega a acumular no es suficiente como para que se generen avalanchas de cierta significación.

3. La morfología de la vertiente - cóncava o convexa - es otro factor que puede inhibir o favorecer respectivamente el deslizamiento de la capa de nieve. Algo más complejo puede ser el análisis de la rugosidad (en principio a un incremento de la rugosidad corresponde un mejor anclaje de la nieve y, por tanto, disminuye la posibilidad de una avalancha), pues en esta propiedad inciden elementos tan diversos como la litología - alternancia de materiales lábiles con otros más resistentes que quedan en resalte-, la disposición de las capas a favor o en contra de la pendiente, la existencia de formas de relieve más o menos favorables, o el tipo de vegetación.

4. Este último elemento, la vegetación, es uno de los criterios directos más importantes para la delimitación de zonas probables de aludes. En primer lugar, en la vegetación 
permanecen las huellas del paso de las avalanchas, manifiestas bien en la ausencia total de aquélla cuando la frecuencia del fenómeno es importante, bien en la existencia de una vegetación específica. Pero además, sabemos que el porte y la densidad de la vegetación son determinantes en relación con el desencadenamiento de aludes. Las zonas cubiertas de pasto serán las más favorables, seguidas de las tapizadas por una masa relativamente densa de arbustos. Los sectores de bosque cerrado serán los menos proclives a registrar avalanchas, si bien pueden verse afectados en mayor o menor medida por avalanchas originadas en otras áreas situadas fuera del bosque a cotas superiores. Por último, la incidencia de la orientación de la vertiente respecto al sol se manifiesta básicamente en el tipo de aludes que tienden a desencadenarse: de nieve suelta o polvo en las laderas septentrionales y de nieve húmeda en las meridionales.

La cartografía de zonas probables de aludes, basada en los criterios que acabamos de mencionar, ha sido elaborada a escala 1:40.000. La elección de esta escala ha venido motivada, en primer lugar, por la propia escala de las fotografías aéreas disponibles (aprox. 1:30.000); adicionalmente consideramos que es una escala adecuada para presentar una primera delimitación básica del problema analizado, que puede resultar, además, de gran utilidad de cara a la realización posterior de cartografías de riesgos. Los elementos con plasmación espacial recogidos en este mapa son los siguientes: 1) zonas probables de aludes; 2) canales de aludes (peligro localizado); 3) conos de origen mixto (de aludes, torrenciales, de gravedad; 4) límites de valle glaciar y/o circos glaciares; 5) escarpes; 6) red hidrográfica; 7) refugios; 8) carreteras; 9) sendas. De entre todos éstos, los dos primeros, constituyen los elementos básicos que siempre aparecen en este tipo de cartografías. En nuestro caso, además, hemos optado por incluir determinados aspectos del relieve que, si bien no aportan información determinante en relación con el tema clave del mapa - la delimitación de zonas probables de aludes-, permiten sin embargo situar el fenómeno de las avalanchas de nieve dentro de un contexto geomorfológico más amplio que puede ayudar a entender su génesis y funcionamiento. Así, por ejemplo, aunque los conos de aludes constituyen zonas que pueden verse afectadas por aludes, dado que en la mayoría de los casos su origen no es exclusivamente nival sino que en él también intervienen la dinámica torrencial y la acción de la gravedad (asociadas también a los canales de aludes), hemos preferido diferenciarlos, añadiendo a la trama utilizada para las zonas de aludes la simbología propia de estas morfologías. Por último, se recogen algunos elementos de origen antrópico (carreteras, sendas y refugios), de manera que, a pesar de que no se trata de una cartografía en la que se contemplen factores como la peligrosidad o los riesgos, al menos la localización de los mismos y su relación con las áreas que pueden verse afectadas por avalanchas puede resultar de cierta utilidad para los gestores o usuarios del espacio analizado.

\section{RESULTADOS}

Tal y como puede apreciarse en la cartografía (Figura 1), una parte importante del valle de Ordesa se halla expuesta a la acción de las avalanchas de nieve. Muchos de los factores 
que se consideran favorables para el desencadenamiento de aludes, anteriormente mencionados, concurren en este sector pirenaico; sector, por otra parte, de gran atracción turística lo que, sin duda, acrecienta el interés de delimitar las áreas más proclives a este tipo de fenómeno. Respecto al primero de los factores, la altitud, ya hemos comentado su papel determinante en la relativa abundancia de precipitaciones en forma de nieve, así como en la conservación del manto nival desde finales de invierno a principios de primavera. Si tomamos como referencia aproximada la cota de $1.670 \mathrm{~m}$ establecida para el valle del Ara (GARCIARUIZ et al., 1985), podemos observar cómo son muchas las zonas que alcanzan una altitud suficiente como para que en ellas se acumule la nieve y se desencadenen aludes; al margen de aquellas otras que, pese a situarse a cotas inferiores, pueden verse afectadas por el paso o la llegada de avalanchas. Igualmente favorables son los valores de las pendientes, pues si consideramos los $28^{\circ}$ como limite inferior requerido para que la masa de nieve supere la componente de fricción de la ladera, tan sólo el propio fondo de valle y algunas zonas limitadas en las proximidades del Tobacor, se encuentran por debajo del mismo; en cuanto a los sectores con pendientes por encima de los $45^{\circ}$, en los que difícilmente se puede acumular nieve suficiente como para dar lugar al desencadenamiento de aludes importantes, coinciden con los grandes escarpes de las paredes del valle. En las vertientes septentrionales fundamentalmente las correspondientes a la Sierra de las Cutas- los cantiles quedan, por tanto, fuera de las áreas que pueden verse afectadas por aludes, iniciándose las mismas a su pie, donde la acumulación de derrubios ha suavizado el perfil de las laderas. Por el contrario, en las vertientes de orientación meridional, hemos englobado estas paredes dado que en la mayor parte de los casos enlazan con zonas de pendientes inferiores en las que sí se acumulan masas importantes de nieve que pueden precipitarse sobre los escarpes.

Dentro del sector cartografiado la litología más favorable en este caso para el desencadenamiento de aludes es la correspondiente a la Formación Flysch. El modelado de los sedimentos tipo flysch (areniscas y margas alternantes en capas decimétricas) se plasma en relieves alomados de suaves pendientes en los que la ausencia de resaltes rocosos facilita el deslizamiento de la nieve a lo largo de importantes trayectorias. Zonas con estas características las encontramos en Salarons y, especialmente, en Punta Tobacor y en Sierra Custodia. Las areniscas de la Formación Tozal configuran los escarpes más abruptos del valle, cuya verticalidad imposibilita la acumulación de nieve. Por otra parte, en los sectores en los que afloran los materiales carbonatados de las Formaciones Salarons y Gallinero abundan los resaltes rocosos y los escarpes de cierta continuidad, elementos que mitigan los efectos de los aludes dado que, en numerosas ocasiones, limitan la extensión máxima de las avalanchas, mientras en las zonas de trayectoria actúan como freno a la energía de la masa de nieve deslizada. También en relación con la rugosidad de las laderas, la escasez o ausencia de vegetación arbórea, que en este sector se manifiesta a partir de los 1.800 $m$ de altitud, constituye uno de los factores más favorecedores. Las comunidades relativamente densas de arbustos difícilmente podrán servir de anclaje una vez que las primeras nevadas los enmascaran, configurando una superficie lisa y deslizante. Los pastizales de herbáceas son la vegetación que presenta una rugosidad más escasa; pero si además se encuentran tapizando las suaves laderas modeladas en el flysch - sector de Tobacor o Sierra Custodia-, constituyen una superficie de deslizamiento excepcional. 


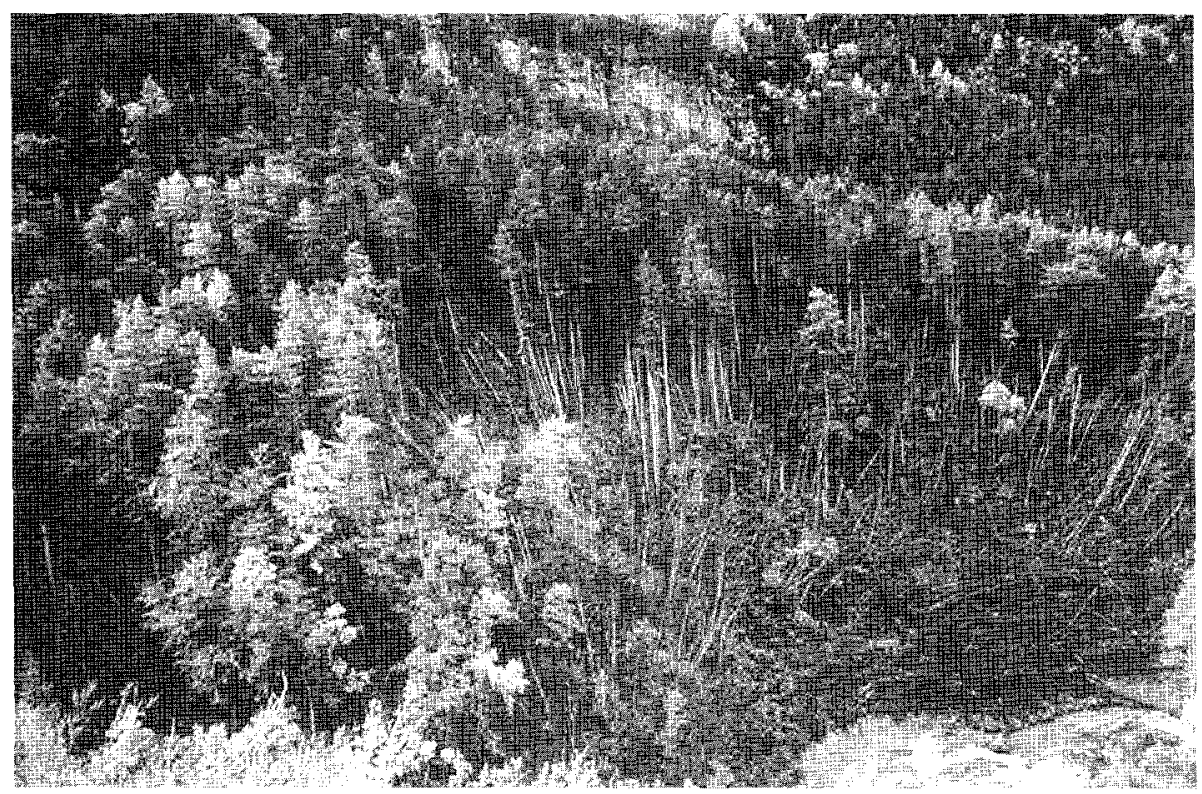

Foto 4. Efectos del alud registrado en el Bco. de la Canal en la temporada invernal 1996-97; el alud atravesó la carretera de acceso a la Pradera de Ordesa y destruyó numerosos pies de pino silvestre situados en la ladera opuesta

En cuanto a la distribución espacial de las zonas que pueden verse afectadas por avalanchas de nieve se aprecia una clara disimetría entre la ladera Norte y la ladera Sur, disimetría que responde fundamentalmente a una diferente morfología entre los dos márgenes del valle. En las vertientes de orientación septentrional, en las que las alturas máximas apenas rebasan los $2.200 \mathrm{~m}$ y en las que los valores de las pendientes son muy elevados, las zonas probables de avalancha —al margen de los canales de aludes, de carácter localizado- conforman una estrecha banda al pie de los grandes cantiles de la Sierra de Las Cutas, banda que tan sólo a partir del sector de las Gradas de Soaso se extiende hasta el fondo del valle. Por el contrario, en las laderas de orientación Sur estas zonas alcanzan una extensión más amplia. En este caso, la mayor elevación de las cotas superiores (por encima de los $2.700 \mathrm{~m}$ en determinados puntos) y, fundamentalmente, el carácter más tendido de las vertientes, favorecen una acumulación de nieve netamente superior.

Los canales de aludes pueden aparecer englobados dentro de áreas probables de aludes, pero muy frecuentemente sirven de conexión entre estas últimas, que constituirían el sector de arranque de los aludes, y el fondo del valle, en el que se ubican los depósitos de origen mixto nivo-fluvial con morfología en conos de dimensiones muy variables. Los ejemplos más significativos de este tipo de canales los encontramos en ambos márgenes del valle, atravesando los bosques mixtos de coníferas y hayas, siendo fácilmente identificables mediante la observación de las alteraciones que sufre la vegetación. Sin embargo, también 
en este caso se aprecia una serie de claras diferencias entre la ladera de orientación Norte y la de orientación Sur. Los caminos de avalancha situados al pie de la Sierra de Las Cutas y que alcanzan el fondo del valle son más numerosos que los que arrancan en el sector de Mondarruego-Punta Escuzana-Salarons y, dado que la pendiente de las vertientes es superior, los aludes que circulen por ellos serán relativamente más frecuentes. Ahora bien, muchos de los canales de las laderas meridionales presentan una longitud mayor y en su cabecera conectan con zonas en las que se acumula una gran cantidad de nieve, lo que significa que en caso de que se desencadene un alud, la peligrosidad será muy superior a la de los aludes que descienden por los canales ubicados en la ladera Norte. Como evidencia de la gran energía que llega a generar una avalancha de nieve canalizada en las vertientes meridionales de Ordesa podemos volver a mencionar el impresionante alud que en el invierno de 1996-97, tras pasar sobre la carretera de acceso a las Praderas de Ordesa, rebasó el fondo del valle, arrasando un sector del bosque de pino silvestre emplazado en la ladera opuesta. La larga trayectoria del canal por el que circuló, conectado con la amplia cuenca modelada al pie de Punta Escuzana, explicarían la magnitud alcanzada por el alud.

\section{CONCLUSIONES}

La observación de fotografías aéreas, complementada con el trabajo de campo y la realización puntual de encuestas, nos ha permitido elaborar una cartografía de zonas probables de aludes del valle de Ordesa. En esta cartografía se incluyen no sólo aquellas áreas en las que tenemos constancia se han registrado avalanchas de nieve, sino también aquellas que, considerando diversos criterios como el límite altitudinal, el valor de las pendientes, la morfología y rugosidad de las laderas, o la vegetación, podrían verse afectadas en algún momento.

Los resultados obtenidos nos muestran que estas zonas son considerablemente extensas, de forma especial en las vertientes de orientación meridional, en las que las condiciones topográficas favorecen la acumulación de un importante manto nival durante los meses de invierno y principios de primavera. Pero además, al margen de estas zonas hemos de destacar los numerosos canales de aludes existentes, muchos de los cuales conectan dichas zonas con el fondo del valle. En este caso, el peligro es de carácter localizado, dado que la nieve se desliza confinada dentro del canal, por lo que la delimitación de la zona que puede verse afectada es mucho más sencilla. Los efectos observados en la vegetación muestran la capacidad destructiva de los aludes que circulan de forma confinada, por lo que resulta de gran interés una localización precisa de los mismos.

La elaboración de cartografías de peligrosidad o de riesgos implicaría en primer lugar considerar los factores meteorológicos, cambiantes a lo largo del tiempo, pero igualmente determinantes, y además combinar la información aportada por la cartografía de zonas probables de aludes con datos relativos a la frecuencia del fenómeno, así como contemplar 


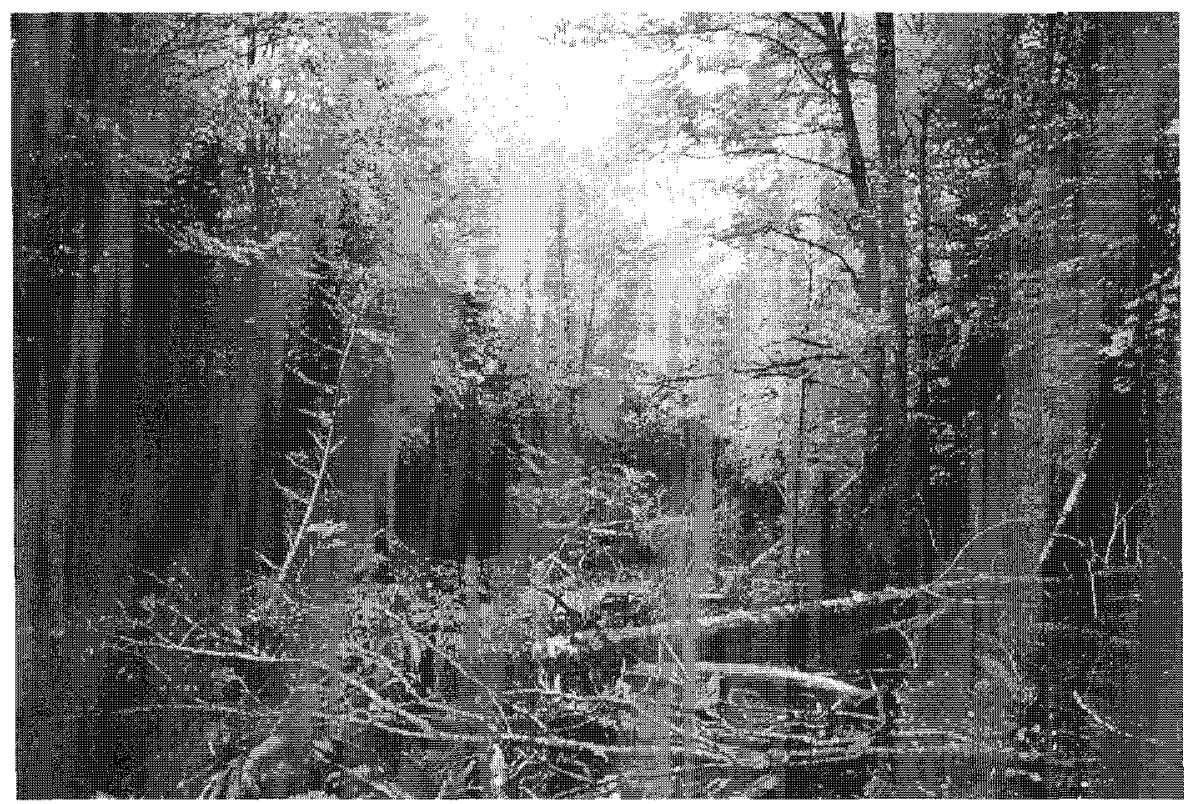

Foto 5. Interior de un canal de aludes al pie de la Sierra de las Cutas, en un sector de bosque mixto próximo al fondo del valle de Ordesa. Obsérvese el alto poder destructivo de estos aludes de corta trayectoria, pero muy repetidos en el tiempo

aspectos antrópicos como la localización de infraestructuras, efectuar valoraciones económicas y valoraciones de peligro directo para el hombre. En cualquier caso, la simple inclusión de los elementos de origen antrópico que aparecen en el valle de Ordesa (vinculados en este caso fundamentalmente a la actividad turística: carreteras, caminos, sendas y refugios), dentro de la cartografía de zonas probables de aludes que hemos realizado, supone ya contar con una herramienta excepcional de cara a la planificación y ordenación de éste y de otros territorios de la montaña aragonesa.

\section{BIBLIOGRAFÍA}

BALCELLS, E. y GIL-PELEGRÍN, E. (1992): «Consideraciones fenológicas de la biocenosis de altitud en el Parque Nacional de Ordesa y Monte Perdido, acompañadas y apoyadas mediante estudio preliminar de los datos meteorológicos obtenidos, desde 1981 a 1989, en el observatorio de Góriz», Lucas Mallada, 4. 71-162, Huesca.

CEMAGREF (1981): Plan de zones exposées atux avalanches. Ministère de l'Agriculture, France. 
FURDADA, G. (1996): Estudi de los allaus al Pirineu Occidental de Catalunya: predicció espacial i aplicacións de la cartografia. Geoforma Ediciones. 315 p. Logroño.

FURDADA, G., VILAPLANA, J. M. y BOSCH, X. (1989): «Predicción de zonas con peligro de alud. Consecuencias socioeconómicas de la falta de planificación territorial». En Fundación MAPFRE/ITSEMAT (Eds.): Encuentro Internacional «Catástrofes y Sociedad». 249-264, Madrid.

GARCÍA-RUIZ, J. M. y MARTÍ BONO, C. E. (1993): Mapa Geomorfológico del Parque Nacional de Ordesa y Monte Perdido. Instituto Pirenaico de Ecología, 119 p. Zaragoza.

GARCÍA-RUIZ, J. M., PUIGDEFÁBREGAS J. y MARTÍ BONO, C. E. (1985): Los recursos hídricos superficiales del Alto Aragón. Instituto de Estudios Altoaragoneses, 224 p. Huesca.

McCLUNG, D. y SCHAERER, P. (1996): Avalanchas. Sua Edizioak-Ediciones Desnivel, 309 p.

RIOS, L. M., GALERA, J. M., BARETTINO, D. y BARNOLAS, A. (1989): Mapa Geológico de España, escala 1:50.000, Hoja 146, Bujaruelo. Instituto Geológico y Minero de España, 64 p. Madrid.

VAN DE VELDE, E. J. (1967): «Geology of the Ordesa overthrust mass, Spanish Pyrenees, province of Huesca». Estudios Geológicos, 23, 167-201, Madrid.

VILLAR, L., SESÉ, J. A. y FERRÁNDEZ, J. V. (1997): Atlas de la Flora del Pirineo Aragonés, Vol. I. Consejo de Protección de la Naturaleza de Aragón-Instituto de Estudios Altoaragoneses, 648 p. Huesca. 